Recepción: 19 / 11 / 2018

Aceptación: 28 / 12 / 2018

Publicación: 20 / 02 / 2019

Ciencias de la salud

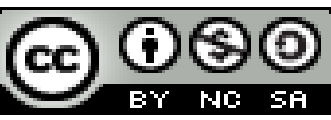

Artículo de Revisión

\title{
Calidad de asistencia dentro de los cuidados de intervención quirúrgica
}

\section{Quality of assistance within the care of surgical intervention}

\section{Qualidade da assistência no cuidado da intervenção cirúrgica}

Pedro F. Delgado-Lino I

pferdelno@gmail.com

Castulo L. Moreira-Moreira II

castuloleonel@hotmail.com

Romulo A. Muñoz-Moreira III

romuloargenis89@gmail.com

Jose F. Macias-Riera IV

freddyjr01@hotmail.com

Gabriela A. Almeida-Almeida V

gaby11031986@gmail.com

Guido Y. García-Loor VI

guido_g191@hotmail.com

Correspondencia:pferdelno@gmail.com

I. Médico Cirujano; Administrador Técnico de Unidad Operativa Tipo A Pacayacu, Sucumbíos, Ecuador.

II. Médico General en Funciones Hospitalarias en el Servicio de Cirugía Hospital Verdi Cevallos Balda de Portoviejo, Ecuador.

III. Médico en funciones Hospitarias; Residente Hospital básico de Flavio Alfaro, Manabí, Manta, Ecuador.

IV. Médico Cirujano; Médico General Asistencial de Cirugía Hosp. Dr. Verdi Cevallos B. Portoviejo, Ecuador.

v. Médico Cirujano; Médico General Asistencial de Cirugía del Hospital Provincial Verdi Cevallos Balda Portoviejo, Ecuador.

vi. Médico Cirujano; Médico General Asistencial; Hospital Provincial Verdi Cevallos Balda Portoviejo, Ecuador. 


\title{
Resumen
}

El estudio se basó en conocer la calidad de asistencia dentro de los cuidados de intervención quirúrgica, a partir del hecho de que las intervenciones médicas pueden ocasionar daños y por lo tanto la calidad. Al momento se disponen de nuevas herramientas y destrezas de administración que otorgan y dirigen el poder a las instituciones de salud que tienen la importancia orientada hacia la calidad asistencial, esta es una herramienta capaz de reconocer, estudiar, cambiar y aplicar sobre los cimientos de una nueva actitud con relación a las efectos adversos como una oportunidad de cambiar y hacer mejor las cosas en cuanto a calidad. Con el desarrollo de este campo cumpliendo con los indicadores de calidad es que va a permitir cumplir con el mandato ético no tan sólo de no dañar a nuestro paciente sino de tratar de beneficiar en todo aquello que el conocimiento y la práctica clínica permite proteger preventivamente tanto al paciente como a los profesionales y concomitantemente a la institución de salud de los juicios de mala praxis. Cabe destacar que la seguridad del paciente dentro de los servicios de salud es una responsabilidad profesional implícita en el acto del cuidado de intervención quirúrgica esto debe estimular a preparar y capacitar enfermeros o enfermeras que tengan una visión de su profesión como disciplina científica que se preocupe fundamentalmente de la humanidad.

Palabras claves: Calidad Asistencial; Nueva Actitud; Mandato Ético; Práctica Clínica; Mala Praxis; Acto del Cuidado.

\begin{abstract}
The study was based on knowing the quality of care within the care of surgical intervention, based on the fact that medical interventions can cause damage and therefore quality. At the moment there are new tools and management skills that grant and direct the power to health institutions that have the importance oriented towards the quality of care, this is a tool capable of recognizing, studying, changing and applying the foundations of a New attitude in relation to adverse effects as an opportunity to change and do things better in terms of quality. With the development of this field, complying with the quality indicators is that it will allow us to comply with the ethical mandate not only to not harm our patient but to try to benefit in everything that knowledge and clinical practice can preventively protect so much to the patient as to the professionals and concomitantly to the health institution of the malpractice trials. It should be noted that patient safety within the health
\end{abstract}


Pedro F. Delgado-Lino; Castulo L. Moreira-Moreira; Romulo A. Muñoz-Moreira; Jose F. Macias-Riera; Gabriela A. Almeida-Almeida; Guido Y. García-Loor

services is a professional responsibility implicit in the act of surgical intervention care. This should stimulate the preparation and training of nurses who have a vision of their profession as a scientific discipline that is fundamentally concerned of humanity.

Keys words: Quality of Care; New Attitude; Ethical Mandate; Clinical Practice; Malpractice; Act of Care.

\section{Resumo.}

O estudo baseou-se em conhecer a qualidade do cuidado dentro do cuidado da intervenção cirúrgica, baseado no fato de que intervenções médicas podem causar danos e, portanto, qualidade. No momento existem novas ferramentas e habilidades de gestão que outorgam e direcionam o poder para as instituições de saúde que têm a importância voltada para a qualidade do cuidado, é uma ferramenta capaz de reconhecer, estudar, mudar e aplicar os fundamentos de uma Nova atitude relação aos efeitos adversos como uma oportunidade para mudar e fazer as coisas melhor em termos de qualidade. Com o desenvolvimento deste campo, cumprir os indicadores de qualidade é que nos permitirá cumprir com o mandato ético não só de não prejudicar o nosso paciente, mas de tentar beneficiar em tudo o que o conhecimento e a prática clínica podem proteger preventivamente tanto para o paciente quanto aos profissionais e concomitantemente à instituição de saúde dos ensaios de malversação. Deve-se ressaltar que a segurança do paciente dentro dos serviços de saúde é uma responsabilidade profissional implícita no ato de atendimento da intervenção cirúrgica. Isso deve estimular a preparação e a formação de enfermeiros que tenham uma visão de sua profissão como uma disciplina científica que é fundamentalmente voltada para a humanidade.

Palavras chaves: Qualidade do cuidado; Nova atitude; Mandato Ético; Prática clínica; Imperícia; Ato de cuidado.

\section{Introducción.}

La calidad del servicio de asistencia en salud así como la seguridad de los cuidados de intervención quirúrgica del paciente ha sido de preocupación constante en las instituciones prestadoras de cuidados tanto como del medio académico, puesto que una asistencia no planificada puede terminar con daños considerables (Paranaguá, Bezerra, Moreira, Tobias, \& Silva, 2016, pag. 229). Por lo 

Pedro F. Delgado-Lino; Castulo L. Moreira-Moreira; Romulo A. Muñoz-Moreira; Jose F. Macias-Riera; Gabriela A. Almeida-Almeida; Guido Y. García-Loor

tanto es de considerar como fundamento esencial y necesario, la buena calidad de asistencia sanitaria y debe estar basada en una actitud preventiva.

Según refieren Borel \& Sivanto (2008) en 1910 Abraham Flexner puso en evidencia la mala calidad en la formación médica de los EEUU, la que constituyó la primera muestra sobre el control de la calidad en los servicios médicos y que en 1916, Codman estableció las bases sobre la importancia de la certificación de los médicos y la acreditación de hospitales, como resultado, en 1918 se creó el Programa de Estandarización de Hospitales, el cual solamente aprobó a 90 de 692 instituciones (Sequeira Cruz, 2016, pag. 10).

Los aportes de pruebas sobre riesgos posibles producidos en contextos diversos han sido numerosos, y desde los decenios de 1950 y 1960 se han realizado estudios que han dado cuenta del problema, aun cuando en ese tiempo no se le dio la dimensión que éste supone. En 1991 el resultado del Harvard Medical Practice Study indicó que un 4\% de los pacientes sufre algún tipo de daño en el hospital; el 70\% de eventos adversos produce incapacidad temporal y el 14\% de los incidentes son mortales $^{3}$ (Cantillo, 2007).

La Organización Mundial de la Salud (OMS) estima que ocurren cerca de 234 millones de cirugías en el mundo durante el año y, debido a ese alto índice de cirugías es importante evaluar la asistencia al paciente durante el periodo perioperatorio² (Paranaguá, Bezerra, Moreira, Tobias, \& Silva, 2016, pag. 229).

En los países tercermundistas y en los que tienen economías en transición (países de Europa del Este) Hungría, Polonia, Rusia, etc., existen pruebas de que la probabilidad en la aparición de eventos adversos son ocasionadas por el mal estado de las infraestructuras, de los equipos, la calidad de los medicamentos y la irregularidad en el suministro, las deficiencias en la eliminación de desechos y en 
el control de las infecciones, la deficiente actuación del personal por falta de motivación o conocimientos insuficientes o número insuficiente y por la falta grave de recursos para cubrir los costos de funcionamiento esenciales ${ }^{2}$ (Cantillo, 2007, pág. 117).

Las intervenciones para el cuidado de la salud del ser humano, en los ámbitos hospitalarios y ambulatorios, están implicando riesgos a partir de la conjugación compleja de procesos, tecnologías e interacciones humanas, que si bien contribuyen en acciones beneficiosas, también incluyen un abanico de posibilidades de práctica insegura para la ocurrencia de eventos adversos (Cantillo, 2007, pag. 113).

En el contexto del mundo globalizado, donde la ciencia, la tecnología y la información están dentro del alcance de muchos, las profesiones, y específicamente la enfermería, enfrentan la necesidad de perfeccionar sus procesos de trabajo con vistas a garantizar cuidados con calidad a los pacientes ${ }^{1}$ (Santana, Bauer, Minamisava, Queiroz, \& Gomes, 2014, pag. 455).

La seguridad de los pacientes ha sido una preocupación de los profesionales de enfermería y ha estado implícita en el acto de cuidar de enfermería, aunque hasta los últimos años se ha identificado como un término novedoso. La presencia de la enfermera en el cuidado, la continuidad del mismo y las condiciones específicas y distintivas de este son elementos centrales para la atención de la salud de las personas en cualquier nivel de la organización, ya que es a través del cuidado de enfermería que se otorga protección y bienestar, así como un ambiente libre de riesgos. Florence Nightingale al institucionalizar la enfermería identificó los elementos necesarios para el proceso de cuidar, entre ellos el medioambiente hospitalario, así como los valores profesionales para la enfermería, tales como no causar daño, el esmero y la dedicación al servicio de las personas, lo que hoy por hoy se 
Pedro F. Delgado-Lino; Castulo L. Moreira-Moreira; Romulo A. Muñoz-Moreira; Jose F. Macias-Riera; Gabriela A. Almeida-Almeida; Guido Y. García-Loor

puede encontrar dentro de los elementos conceptuales para la seguridad del paciente (ZárateGrajales, y otros, 2015, pag. 65).

La literatura describe que, en países desarrollados, parte de las muertes no esperadas se generan a partir de incidentes relacionados con la seguridad del paciente, lo cual genera una alerta en la atención hospitalaria, a la vez que el error clínico también provoca complicaciones no esperadas. Esto puede conllevar efectos no reversibles como la muerte o efectos secundarios con alto costo tanto para el afectado como para las instituciones de salud (Ramos, Coca, \& Abeldaño, 2017, pag. 49).

Para enfermería la seguridad es intrínseca al cuidado y a la calidad de este, ya que implica satisfacer las necesidades y cumplir con las expectativas de las personas y sus familias, con la premisa clara de no hacer daño. Al evaluar la calidad y seguridad de la atención, se deberá considerar como indicador los eventos adversos (EA), a partir de conocer la esencia de estos, ya que no siempre son resultado de fallas en los procesos de atención en sus diferentes dimensiones, es decir, no necesariamente derivan de un fallo humana sino que pueden presentarse por aspectos propios del sistema de salud. Así, hablar de un cuidado seguro implica una práctica profesional basada en evidencia, intencionada, planeada, que proteja y ayude a las personas, creadora de condiciones favorables en el medio ambiente del sujeto de cuidado (Zárate-Grajales, y otros, 2015, pag. 65).

La Organización Mundial de la Salud alerta de que millones de personas en el mundo sufren lesiones incapacitantes y muertes derivadas de prácticas sanitarias que no son seguras. De acuerdo con lo anterior, se estima que uno de cada 100 pacientes será víctima de un error. Esa estimación viene de estudios conducidos en países desarrollados, con sistemas de salud más estructurados que los de países en desarrollo, en los cuales los datos sobre la seguridad de los pacientes como parte de 
la gestión de calidad aún son escasos. En los Estados Unidos, se considera que 100 personas mueren diariamente debido a errores ocurridos durante la asistencia sanitaria, cifra que convierte el error en la octava causa de mortalidad (Ramos, Coca, \& Abeldaño, 2017, pag. 49).

La enfermería es una profesión con profundas raíces humanistas, genuinamente preocupada por las personas que confían en su cuidado, en especial de quienes experimentan una situación de enfermedad y sufrimiento. Es una actividad formal que aprecia y valora la conciencia colectiva del gremio. Sus fundamentos dan sustento a una práctica, profundamente humanista, que ha evolucionado con el avance científico-técnico. Acorde con su propósito de superación, se acoge a la corriente que tiende a garantizar la calidad en los sistemas de los servicios de salud, que busca satisfacer las necesidades de la sociedad. Así, la calidad se ha convertido en un elemento esencial de los servicios de salud1 (León Román, 2006, pag. 1).

Los servicios sanitarios son de calidad cuando carecen de deficiencia y satisfacen las necesidades del usuario interno y externo. Una asistencia sanitaria de calidad es la aplicación correcta, en la asistencia a los pacientes, de los principios esenciales de las ciencias médicas, al mismo tiempo que se equiparan los riesgos inherentes a cada actividad asistencial realizada a los beneficios que se esperan de ella ${ }^{6}$ (Minchón Hernando, y otros, 2011, pag. 299).

En los últimos tiempos, el paciente se ha convertido en el principal centro e impulso para elevar la calidad de los servicios sanitarios, por lo que su grado de satisfacción es el indicador fundamental de la calidad asistencial ${ }^{1}$ (Minchón Hernando, y otros, 2011, pag. 299).

Actualmente, los servicios de salud enfrentan numerosos desafíos para conseguir atender las demandas de los clientes y familiares, con miras a la excelencia de la calidad asistencial. Para alcanzar esta excelencia, los profesionales de salud deben asumir un proceso de trabajo con foco en 
Pedro F. Delgado-Lino; Castulo L. Moreira-Moreira; Romulo A. Muñoz-Moreira; Jose F. Macias-Riera; Gabriela A. Almeida-Almeida; Guido Y. García-Loor

la mejora continua, favoreciendo la identificación constante de los factores intervinientes en la asistencia y la elaboración de instrumentos que posibiliten evaluar sistemáticamente los niveles de calidad prestados ${ }^{6}$ (Paranaguá, Bezerra, Moreira, Tobias, \& Silva, 2016, pag. 230).

\section{Metodología.}

El presente trabajo tiene una metodología de tipo bibliográfica. Ya que se ha cumplido con una revisión de literatura en diferentes bases de datos, libros electrónicos, consultas electrónicas, artículos científicos y revistas. Para lo cual se consideró, información confiable sobre exploraciones versadas en este tema, en los que se estudia la calidad de asistencia dentro de los cuidados de intervención quirúrgica.

\section{Resultados.}

\section{Calidad asistencial en el paciente quirúrgico}

Cualquier acto quirúrgico, por pequeño que sea, es una fuente potencial de estrés que trae aparejada respuestas psicológicas y fisiológicas, las que pueden sin duda comprometer la colaboración del paciente, el seguimiento de las prescripciones médicas, la efectividad del proceder y el proceso de recuperación (Amador, 2010, pag. 53).

La calidad en salud se define como la clase de asistencia que se espera que aumente al máximo una medida incluida del bienestar del paciente, después de tener en cuenta el equilibrio de los esperados beneficios y pérdidas que concurren en el proceso de la asistencia en todos sus componentes: técnico, interpersonal así como físico y social (González Pérez, Fernández Clúa, \& Jiménez Serrano, 2011, pag. 5). 
El principio "Primum Non Nocere" subyace a cada acto asistencial, por lo que podríamos asumir como principio básico que cada profesional es un sujeto competente y responsable que tiene como base de su actuación no generar daño. A pesar de ello, y por la cada vez mayor complejidad de la atención, el componente humano individual interactúa con varios factores. Elementos propios del paciente, de las tareas o procedimientos, de la estructura físico ambiental que incluye el material, el equipamiento técnico y el lugar físico donde se produce la asistencia o de la organización y que es causa de que los eventos adversos puedan presentarse de forma más frecuente de lo deseado (ROCCO \& GARRIDO, 2017, pag. 786).

Es fácil comprender el impacto de la palabra calidad si se considera su origen del griego Kalos que significa lo bueno, lo hermoso, apto, favorable y del latín Qualitatem (propiedad)1 (González Pérez, Fernández Clúa, \& Jiménez Serrano, 2011, pag. 4).

Según González Steinbauer, (2015) los componentes esenciales de la calidad asistencial son tres:

a) El factor científico-técnico: La aplicación del mejor conocimiento disponible en función de los medios y recursos disponibles.

b) El factor percibido: La satisfacción de los pacientes con la atención, los resultados y el trato recibido.

c) El factor institucional o corporativo: Ligado a la imagen de un centro sanitario (p. 8-9).

En el estudio de Aiken, et al., identificaron que la formación de enfermería es importante para la calidad del cuidado y la mejora en el resultado para el paciente; por ejemplo, cada aumento del 10\% de enfermeras contratadas con licenciatura se asocia a una disminución de mortalidad del paciente en un 5\%, de ahí el interés por la preparación adecuada de las enfermeras, ya que de ellas depende 
Pedro F. Delgado-Lino; Castulo L. Moreira-Moreira; Romulo A. Muñoz-Moreira; Jose F. Macias-Riera; Gabriela A. Almeida-Almeida; Guido Y. García-Loor

en gran medida la prevención y la identificación de riesgos relacionados con la seguridad a partir de proveer cuidados de calidad dentro de los diversos procesos (Zárate-Grajales, y otros, 2015, pag. 70).

El estándar es el valor que toma un indicador y que separa el límite entre lo aceptable y lo inaceptable, o lo que es igual, pone la frontera entre lo que se considera una práctica de calidad de otra de no-calidad o inaceptable. Los estándares se pueden fijar por comités de expertos, por frecuencias intermedias entre hospitales, por valores bibliográficos, pero deben ser aceptados por todos aquellos que vayan a ser valorados y nunca ser impuestos por la fuerza. Estos se pueden diferenciar en: estándares óptimos, aceptables y subóptimos $^{9}$ (González Pérez, Fernández Clúa, \& Jiménez Serrano, 2011, pag. 6).

La causa más común de los errores no está en los individuos de forma particular, sino en las fallas del sistema, los procesos y las condiciones que llevan a la gente a cometer errores o fallas en la prevención de los mismos, los cuales convergen en la formación, capacitación y entrenamiento (Zárate-Grajales, y otros, 2015, pag. 70).

Según Rojas (2001) citado por Contreras \& Ensayo (2005), la gestión clínica, entendida como el conjunto de procesos implicados en la relación entre ese profesional y paciente, puede articularse en tres niveles diferenciados:

- Nivel individual, referido a la elección de un determinado tratamiento o intervención quirúrgica de mayor eficacia. Para tal efecto, se utilizan herramientas como medicina basada en la evidencia, guía de práctica clínica, epidemiología, auditorias, compromisos de gestión.

- Nivel asistencial, relativo a la efectividad clínica de los procesos y recursos empleados. 
- Nivel de gestión de la unidad clínica; vinculado a eficiencia, la organización interna del servicio, el número de personas considerado necesario para cada tipo de actividad o técnica y los aspectos más globales como la motivación y capacitación del personal, así como la gestión de los recursos asignados (p. 26).

Según Arnal (2001), el o la profesional en Enfermería toma todas las decisiones, en momentos diversos, con el paciente o en su nombre; por eso, incide en forma directa en el proceso para optimizarle la calidad de vida. Parece absolutamente razonable que, si se pretende mejorar los resultados, el profesional se incorpore a la gestión de los recursos de las unidades clínicas (Contreras \& Ensayo, 2005, pag. 26).

Según Cortés (2003), la gestión solo se logra mejorando la eficacia, la eficiencia y la calidad, mediante el uso de protocolos basados en la evidencia científica, en los procedimientos de asistencia; además, facilitando la implicación de los profesionales en la gestión, mediante el incremento de su intervención en la toma de decisiones e incentivando el esfuerzo y los resultados, tanto en su competencia clínica como en su gestión y desarrollo de la promoción profesional (Contreras \& Ensayo, 2005, pag. 25).

En relación a calidad en salud, debemos indicar que en un principio prevalecía la opinión de que cada persona tenía derecho a la mejor medicina que se la puede ofrecer; gradualmente se han agregado algunos componentes al concepto de Calidad Total. Uno de ellos es la satisfacción tanto del que proporciona el servicio, del que lo recibe y de la entidad responsable de que se brinden los servicios; si a esto le agregamos los recursos y los costos, estará completo el escenario en que se conceptúa la Calidad Total (De Lille, 2013, pag. 71). 
Pedro F. Delgado-Lino; Castulo L. Moreira-Moreira; Romulo A. Muñoz-Moreira; Jose F. Macias-Riera; Gabriela A. Almeida-Almeida; Guido Y. García-Loor

Según De Lille-y Fuentes (2013), la Calidad Total, busca la satisfacción, permanente de las necesidades del paciente; por lo tanto, el hospital debe fundamentalmente conocer cuáles son esas necesidades y diseñar en base a las mismas una atención que se ajuste a sus requerimientos, de lo contrario el paciente puede optar por el cambio. El concepto de Calidad Total en salud tiene tres principios básicos:

- Todo esfuerzo debe estar dirigido al paciente.

- Nada es mejor que trabajar en equipo de salud.

- Todo lo que hacemos es susceptible de mejorar (p. 71).

La creación de una cultura de calidad total constituye un nuevo reto para todo el personal de salud en los tiempos actuales, pues representa un cambio profundo en la filosofía de las instituciones que brindan salud y es una demanda creciente para poder sobrevivir en un mundo cada vez más competitivo (De Lille, 2013, pag. 74).

\section{Cuidados en el paciente de intervención quirúrgico}

La comunicación de una intervención quirúrgica representa un alto nivel de estrés en el plano emocional, lo que se incrementa si se trata de cirugías del corazón o del cerebro, asociadas históricamente con la muerte. Son múltiples las reacciones psicológicas que, ante un evento como este, pueden desencadenarse, vinculadas con el temor al dolor, a lo desconocido, a su reacción a la anestesia, al propio proceder, a perder la integridad del cuerpo o la autonomía e incluso a morir (Amador, 2010, pag. 53).

El proceso de enfermedad en general y la cirugía en particular, junto con la falta de control del entorno expone a la persona enferma a una importante situación de vulnerabilidad física y 
psicológica. En esta circunstancia los cuidados de enfermería cobran una especial relevancia y obligan a proporcionar unos cuidados excelentes desde el ingreso, dirigidos a garantizar su seguridad, mantener la dignidad de la persona y prevenir complicaciones (Vallejo, Becerra, \& Hervás, 2007, pag. 4).

El abordaje por enfermería sobre temas de calidad y efectividad de los tratamientos en los pacientes, se remonta a tiempos pasados, como se puede apreciar en el postulado de Florence Nightingale, cuando planteó “...las leyes de la enfermedad pueden ser modificadas si comparamos tratamientos con resultados...” (León Román, 2006, pag. 2).

Las intervenciones de atención a la salud se realizan con el propósito de beneficiar a los pacientes, pero también se ha identificado que muchas de ellas, por diversos factores, pueden causarles daño, y prueba de ello es lo que reporta la Organización Mundial de la Salud, ya que calcula que en los países desarrollados hasta uno de cada 10 pacientes sufre algún tipo de daño durante su estancia en el hospital (Zárate-Grajales, y otros, 2015, pag. 68).

Según Benavent, et al. (2000), citado por León Román (2006), en estos momentos en que avanzamos hacia un mayor requerimiento social de nuestros servicios y con ello, consolidar la presencia en un campo fuertemente profesionalizado, a un nivel de colaboración y no de subordinación, se puede plantear que la enfermería es una profesión porque:

- Constituye un servicio a la sociedad.

- Posee un cuerpo de conocimientos y habilidades propias, que busca constantemente acrecentar su competencia para mejorar la calidad de sus servicios.

- Cuenta con un objeto de estudio (el cuidado), razón de ser de la enfermería.

- Establece sus propias normas y políticas para controlar sus actividades. 
Pedro F. Delgado-Lino; Castulo L. Moreira-Moreira; Romulo A. Muñoz-Moreira; Jose F. Macias-Riera; Gabriela A. Almeida-Almeida; Guido Y. García-Loor

- Se encarga de la preparación de las personas que van a desempeñar la profesión.

- Adapta sus servicios a las necesidades que se le van presentando.

- Acepta la responsabilidad y compromiso de proteger al público al que sirve.

- Trata de utilizar al máximo de sus posibilidades, a las personas que la ejerce.

- Regula su propia práctica.

- Se ajusta a un código de conducta basado en sus principios éticos.

- Convoca la unión de sus miembros con el propósito de alcanzar objetivos comunes en respuesta al encargo social (p. 2-3).

La evaluación de la satisfacción del paciente ha sido adoptada por las instituciones de salud como una estrategia para alcanzar un conjunto de percepciones relacionado a la calidad de la atención recibida, con el cual se adquiera informaciones que benefician la organización de esos servicios ${ }^{10}$ (Santana, Bauer, Minamisava, Queiroz, \& Gomes, 2014, pag. 455).

La enfermería abarca los cuidados autónomos y en colaboración que se prestan a las personas de todas las edades, familias, grupos y comunidades, enfermos o sanos, en todos los contextos, e incluye la promoción de la salud, la prevención de la enfermedad, y los cuidados de los enfermos, discapacitados, y personas moribundas. Funciones esenciales de la enfermería son la defensa, el fomento de un entorno seguro, la investigación, la formación, la participación en la política de salud y en la gestión de los pacientes y los sistemas de salud (Perez Ibarra, 2008).

La enfermería tiene conciencia de su responsabilidad ante la calidad del cuidado que presta al paciente, a la institución, a la ética, a las leyes y a las normas de la profesión, y también de la contribución de su desempeño en la valuación del cuidado y satisfacción de los pacientes ${ }^{12}$ (Santana, Bauer, Minamisava, Queiroz, \& Gomes, 2014, pag. 455). 
Los principales componentes del cuidado que hacen posible alcanzar los objetivos son: carácter tangible, fiabilidad, rapidez, competencia, cortesía, credibilidad, seguridad, accesibilidad, oportunidad, comunicación y conocimiento de la situación del paciente (León Román, 2006, pag. 5).

León (2006), plantea que la seguridad del paciente dentro de los programas de garantía de calidad, no es un modismo, ni un nuevo enfoque en los servicios de salud; sino una responsabilidad profesional implícita en el acto del cuidado. Se debe preparar enfermeros que tengan una visión de su profesión como disciplina científica, que se preocupen por el bien fundamental de la humanidad y que respondan a su encargo social a brindar cuidados oportunos, con calidad y libres de riesgo (p. $6)$.

La enfermería, al brindar su cuidado a personas con problemas de salud, debe tener sus acciones dirigidas a las demandas biológicas, sociales, espirituales y psíquicas del ser humano. Para estimular y ayudar las personas a satisfacer sus necesidades espirituales, el profesional de enfermería puede valerse de algunas intervenciones, tales como: ofrecimiento de apoyo a la necesidad espiritual o religiosa, facilitación de la práctica de una religión, aconsejarles espiritualmente, contactar un consejero espiritual y ayudar en la resolución entre el tratamiento y las creencias espirituales (Santana, Bauer, Minamisava, Queiroz, \& Gomes, 2014, pag. 458).

Oír lo que los pacientes tienen para relatar sobre el cuidado que le es prestado y sobre su satisfacción puede ser una oportunidad de construcción de un indicador de resultado, que indica a los gestores algunos caminos decisorios de transformaciones e innovaciones. Cambios en el contexto mundial han hecho con que los pacientes y sus familiares exijan un comprometimiento renovado con la mejora de la calidad en organizaciones prestadoras de servicios de salud, 
Pedro F. Delgado-Lino; Castulo L. Moreira-Moreira; Romulo A. Muñoz-Moreira; Jose F. Macias-Riera; Gabriela A. Almeida-Almeida; Guido Y. García-Loor

estimulados por la preocupación con la seguridad del paciente (Santana, Bauer, Minamisava, Queiroz, \& Gomes, 2014, pag. 455).

Respecto a la calidad del cuidado prestado por el equipo de enfermería según la perspectiva del paciente, se pudo concluir que hay un déficit de la calidad de los cuidados de enfermería prestados en la institución, y también un alto nivel de satisfacción de los pacientes con los cuidados de enfermería recibidos, en todos los dominios evaluados. Sin embargo, se identificó una correlación débil a moderada entre calidad de la atención de enfermería y satisfacción del paciente (Santana, Bauer, Minamisava, Queiroz, \& Gomes, 2014, pag. 459).

Los ciudadanos, elemento fundamental sobre el que gira cualquier política de calidad, también de los sistemas sanitarios, deben de ocupar el espacio de participación, autonomía en la toma de decisiones y corresponsabilidad como valores esenciales de la organización sanitaria en particular y de la sociedad en general (Plan de Calidad del SSPA, 2010). Bajo esta perspectiva de participación y a través del análisis de satisfacción, conocemos la percepción que sobre nuestros servicios de cirugía oftalmológica tienen los usuarios. Si bien es cierto que existen muchos estudios sobre satisfacción de pacientes que resaltan la alta consideración de los cuidados-intervenciones ofrecidas por las enfermeras, en base a nuestros resultados, podemos estar de acuerdo con (García, 2008), en que el carácter educativo de la enfermería es fundamental en adultos mayores para asegurar la comprensión y aumentar la seguridad (Minchón Hernando, y otros, 2011, pag. 307).

A fin de disminuir los posibles riesgos de la intervención quirúrgica, el cuidado debe planificarse según la individualidad de cada paciente, basado en evidencias científicas y determinado por la condición clínica del paciente10 (Paranaguá, Bezerra, Moreira, Tobias, \& Silva, 2016, pag. 230). 
Las enfermeras(os) estamos directamente implicadas(os) en la provisión de un ambiente seguro, en el cual las personas puedan desenvolverse sin sufrir daños y en el que perciban una sensación de seguridad. No debemos olvidar que el paciente hospitalizado experimenta una dualidad en tanto que el hospital se convierte en el control de la situación de salud que le aqueja, pero la separación familiar y el cumplimiento de normas que rigen a las instituciones de salud le representan sentimientos de inseguridad ${ }^{14}$ (Cantillo, 2007).

Las nuevas tecnologías en el cuidado de salud han aumentado los costes del sector salud y las expectativas de la población con relación a los servicios ofrecidos. Sin embargo, estudios indican fallas en la calidad y seguridad de la atención, con ocurrencia de eventos indeseados, perjudicando el imagen de las organizaciones de salud (Estepa Largo \& Tipanta Criollo, 2016, pag. 2).

De acuerdo a la Agency for Health Care Research and Quality-AHQR, la cultura de seguridad se define como el resultado de los valores individuales y de grupo, de las actitudes, percepciones, competencias y patrones de comportamiento que determinan el compromiso, el estilo y la competencia de una organización hacia la salud. Además del manejo de la seguridad, que se caracteriza por una comunicación fundada en la confianza mutua, producto de las percepciones compartidas acerca de la importancia de la seguridad, y por la confianza depositada en la eficacia de las medidas preventivas implementadas para evitar los eventos adversos ${ }^{4}$ (Ramos, Coca, \& Abeldaño, 2017, pag. 49).

Persiste una cultura de culpabilización de las personas que cometen un error, cultura en la que los errores son vistos como consecuencias de la desatención o de la falta de competencia. Al respecto, Reason ha teorizado que, en el ámbito de la salud, los errores no solo se producen por un descuido 
Pedro F. Delgado-Lino; Castulo L. Moreira-Moreira; Romulo A. Muñoz-Moreira; Jose F. Macias-Riera; Gabriela A. Almeida-Almeida; Guido Y. García-Loor

personal, sino por un conjunto de fallas secuenciales en las que algunos aspectos estructurales del sistema de salud tienen una gran participación ${ }^{21}$ (Ramos, Coca, \& Abeldaño, 2017, pag. 52).

Según Fernández (2004), complicación evitable, efecto previsible consecuente o relacionado con la gravedad o patología de base. Dentro de esta categoría se encuentran:

- Infecciones intrahospitalarias.

- Desajustes metabólicos.

- Consecutivas a procedimientos instrumentales, quirúrgicos.

- Problemas transfusionales.

- Problemas anestésicos.

- Reinternaciones por la misma patología.

- Reingreso a terapia intensiva.

- Reintervenciones quirúrgicas (p. 404).

Leape, et al. (1993), Citado por Fernández Busso (2004) manifiestan como en un estudio donde se utilizó otro tipo de clasificación según el proceso y el enfoque de la atención. Un grupo de errores que conviene diferenciar es aquel vinculado con la administración de productos medicinales. Son reconocidos los riesgos inherentes a la terapia con fármacos asociados con el uso terapéutico de medicamentos prescriptos y no prescriptos. Estos efectos desfavorables se pueden calificar genéricamente como reacciones adversas y errores de medicación. A continuación, la clasificación de errores según las etapas de la atención: 
Tipo de errores generales

\section{Diagnóstico}

- $\quad$ Error o demora en el diagnóstico.

- Falla en el empleo de las pruebas indicadas.

- Uso de pruebas o terapias antiguas.

- Fracaso de los resultados de monitoreo o pruebas.

\section{Tratamiento}

- Error en el desarrollo de operación, procedimiento o prueba.

- $\quad$ Error en la administración del tratamiento.

- Error en la dosis o método de aplicación de medicamentos.

- Demora de tratamiento o ante una prueba anormal.

- Cuidado inapropiado o no indicado.

\section{Prevención}

- Demora en la provisión de un tratamiento preventivo.

- Inadecuado monitoreo o del seguimiento del tratamiento.

- Fallas en la comunicación.

- Fallas del equipamiento.

- Otras fallas o demoras (p. 404).

Cabe resaltar que una de las complicaciones más frecuentes que ocurre en los pacientes son las infecciones nosocomiales, las cuales pueden llevarlos a la muerte y, por tanto, se han constituido en 
Pedro F. Delgado-Lino; Castulo L. Moreira-Moreira; Romulo A. Muñoz-Moreira; Jose F. Macias-Riera; Gabriela A. Almeida-Almeida; Guido Y. García-Loor

un reto mundial, en busca de la seguridad del paciente. El riesgo de adquirirlas está en relación directa con el estado de salud del paciente cuando ingresa a las instituciones, el número de pruebas diagnósticas y el tratamiento que se realiza durante la intervención. Aquí, cobra importancia reconocer que para explicar el tema de los eventos adversos son dos los criterios utilizados: el humano y el del sistema (Cantillo, 2007, pag. 114).

El primero de ellos, el humano, originado por los actos inseguros y la violación de los procedimientos que el personal del equipo de salud comete debido a cansancio, estrés, falta de motivación, de atención, sobrecarga y/o insatisfacción laboral y negligencia. Es el llamado Síndrome de Burnout, caracterizado por agotamiento, pérdida de energía que experimentan los profesionales cuando sienten que los problemas de los otros los saturan. Este aspecto, que afecta la seguridad de los pacientes, tiene una connotación importante a la hora de velar por el cuidado que se brinda. El ser cuidado observa este comportamiento como una deshumanización del equipo de salud frente a la situación que vive (Cantillo, 2007, pag. 114).

El segundo criterio, el del sistema, explica la susceptibilidad que tiene el ser humano de cometer errores, a pesar de estar en la organización de más alta calidad, y por tanto, lo importante es generar mecanismos de defensa para prevenir errores. Al presentarse el error, lo fundamental es centrarse en la búsqueda de la explicación del cómo y por qué fallaron esos mecanismos de defensa y no en buscar culpables (Cantillo, 2007, pag. 114-115).

"la cirugía segura salva vidas", a partir de la constatación de que un 25\% de las hospitalizaciones quirúrgicas pueden presentar complicaciones, de que cada año ocurren 7 millones de complicaciones incapacitantes y de que entre un 0.5 - 5\% de los pacientes mueren tras la cirugía y ocurren 1 millón de muertes al año en todo el mundo, la OMS considera a la práctica de la cirugía como un asunto de 
salud pública y promueve establecer una serie de estándares mínimos, que pueden ser aplicados universalmente, definiendo un "entorno de seguridad en el proceso quirúrgico", que se concreta en la difusión y aplicación de un "check-list" o listado de verificación quirúrgica. La iniciativa se concentra en cuatro áreas temáticas y en una serie de aspectos esenciales:

1. Cirugía limpia: mediante el lavado de manos, uso apropiado de antibióticos, preparación de la piel, cuidado de la herida quirúrgica y descontaminación del material.

2. Anestesia segura: A través de la presencia de un anestesista entrenado, pulsioximetría y monitorización adecuada de la frecuencia cardiaca, presión sanguínea y temperatura.

3. Equipos quirúrgicos adecuados y profesionales seguros: personal entrenado, identificación inequívoca del paciente y lugar correcto de la cirugía, mecanismos de control del dolor, consentimiento informado, disponibilidad de equipamiento e instrumental.

4. Garantizar la calidad: implantar medidas y mecanismos que aseguren la calidad mediante revisión del seguimiento de las complicaciones y revisiones por expertos.

Autoras como Rosa $\mathrm{M}^{\mathrm{a}}$ Alberdi plantean que dentro de la práctica asistencial registrada, se incorpore "la mirada enferma". La visión enfermera nos permite ver a la persona más allá de la enfermedad, junto a la tarea de vigilar parámetros y administrar la medicación pautada. Nuestra perspectiva permite ver a un ser humano inmerso en un proceso en el que necesita cuidarse y que se le cuide en un entorno determinado, y esto además de vigilar su órgano enfermo. Sobre esta base y para facilitar la ejecución de los cuidados en el periodo perioperatorio, se estructura un mapa de cuidados (MC), partiendo desde el preoperatorio, pasando por el intraoperatorio, postoperatorio inmediato, postoperatorio tardío y alta domiciliaria. MC forma parte del modelo organizativo denominado gestión de cuidados, en el que se integran y coordinan las actuaciones de diferentes profesionales, 
Pedro F. Delgado-Lino; Castulo L. Moreira-Moreira; Romulo A. Muñoz-Moreira; Jose F. Macias-Riera; Gabriela A. Almeida-Almeida; Guido Y. García-Loor

desde el principio de la estancia hospitalaria hasta el fin, para alcanzar los resultados clínicos óptimos (Fernández \& Martín, 2016, pag. 191).

\section{Conclusiones.}

La calidad de la asistencia y los cuidados de intervención quirúrgica en que los médicos, enfermeras y otros profesionales acceden a la información para hacer su trabajo de mejoramiento de hecho modificando la prestación de sus servicios usando el método científico como parte integral de la responsabilidad de toda la organización. Al momento se disponen de nuevas herramientas y destrezas administrativas que otorguen y dirijan el poder a las instituciones de salud que tienen la importancia orientada hacia la calidad asistencial, esta es una herramienta capaz de reconocer, estudiar, cambiar y aplicar sobre los cimientos de una nueva actitud con relación a las efectos adversos como una oportunidad de cambiar y hacer mejor las cosas en cuanto a calidad. Con el desarrollo de este campo cumpliendo con los indicadores de calidad es que va a permitir cumplir con el mandato ético no tan sólo de no dañar a nuestro paciente sino de tratar de beneficiar en todo aquello que el conocimiento y la práctica clínica permite proteger preventivamente tanto al paciente como a los profesionales y concomitantemente a la institución de salud de los juicios de mala praxis. También es importante considerar estrategias dirigidas a mejorar la comunicación con los pacientes y familiares, desde el profesional en salud incluido la enfermería, mejorando la relación enfermerapaciente como parte fundamental de una relación basada en la confianza y seguridad.

\section{Bibliografía.}

Amador, Y. C. (2010). Intervención psicológica en cirugía cardíaca. Avances en psicología latinoamericana, 25(1), 52-63.

Cantillo, E. V. (2007). Seguridad de los pacientes. Un compromiso de todos para un cuidado de calidad. Salud uninorte, 23(1). 
Contreras, Z., \& Ensayo, M. (2005). Desarrollo e implementación de la gestión clínica de profesionales en enfermería. Costa Rica.

De Lille, R. M. (2013). Calidad en anestesia. . Revista Mexicana de Anestesiología, 36(S1), , 69-74.

Estepa Largo, O. P., \& Tipanta Criollo, D. (2016). Estudio de la calidad de la atención de enfermería caso de estudio Servicio de Emergencias Pediátricas del Hospital General Pablo Arturo Suárez de la Ciudad de Quito, en el período noviembre 2015-febrero 2016. Quito.

Fernández Busso, N. (2004). Los eventos adversos y la calidad de atención: Estrategias para mejorar la seguridad de los pacientes pediátricos. Archivos argentinos de pediatría, 102(5), 402-410.

Fernández, R. G., \& Martín, J. (2016). La seguridad quirúrgica en el marco del Sistema Nacional de Salud de España. Revista CONAMED, 15(4).

González Pérez, E., Fernández Clúa, M., \& Jiménez Serrano, D. (2011). Calidad en los servicios médicos en anestesiología: algunas consideraciones actuales. Revista Cubana de Anestesiología y Reanimación, 10(1), 2-11.

González Steinbauer, C. D. (2015). Revisión de las admisiones en Urgencias como elemento evaluador de la Calidad Asistencial. Impacto de los eventos adversos en el Servicio de Urgencias de un hospital Comarcal. Impacto económico de los eventos adversos.

León Román, C. A. (2006). La seguridad del paciente, una responsabilidad en el acto del cuidado. Revista Cubana de enfermería, 22(3), 0-0.

Minchón Hernando, A., Díaz Jiménez, M., Muñoz, C., De Porras Carrasco, R., Domínguez García, G., \& Vázquez de la Rosa, M. (2011). Análisis de satisfacción del paciente quirúrgico en oftalmología. Enfermería Global, 10(23), 298-309.

Paranaguá, T. T., Bezerra, A., Moreira, I., Tobias, G., \& Silva, A. (2016). Indicadores de asistencia en un clínica quirúrgica. Enfermería Global, 15(43), 228-239.

Perez Ibarra, A. (11 de 06 de 2008). Obtenido de https://anibalpi.wordpress.com/2008/06/11/ladefinicion-de-enfermeria-del-cie/

Ramos, F., Coca, S., \& Abeldaño, R. (2017). Percepción de la cultura de seguridad de pacientes en profesionales de una institución argentina. Enfermería universitaria, 14(1), 47-53.

ROCCO, C., \& GARRIDO, A. (2017). SEGURIDAD DEL PACIENTE Y CULTURA DE SEGURIDAD. REV. MED. CLIN. CONDES - 2017; 28(5), 785-795.

Santana, J., Bauer, A., Minamisava, R., Queiroz, A., \& Gomes, M. (2014). Calidad de los cuidados de enfermería y satisfacción del paciente atendido en un hospital de enseñanza. Rev. LatinoAm. Enfermagem, 22(3), 454-60.

Sequeira Cruz, C. A. (2016). Evaluación de la calidad de atención en la prestación de asistencia médica y satisfacción de los pacientes del servicio de anestesiología del Hospital Militar Escuela Dr. Alejandro Dávila Bolaños en Período de Septiembre a Noviembre del año 2015. 
Pedro F. Delgado-Lino; Castulo L. Moreira-Moreira; Romulo A. Muñoz-Moreira; Jose F. Macias-Riera; Gabriela A. Almeida-Almeida; Guido Y. García-Loor

Vallejo, J. C., Becerra, F., \& Hervás, J. (2007). Planificación de cuidados en el paciente quirúrgico. Inquietudes: Revista de enfermería, 13(36), 4-16.

Zárate-Grajales, R., Olvera-Arreola, S., Hernández-Cantoral, A., Corral, S., Sánchez-Angeles, S., Labastida, R., \& Zapién-Vázquez, M. (2015). Factores relacionados con eventos adversos reportados por enfermería en unidades de cuidados intensivos. Proyecto multicéntrico. Enfermería universitaria, 12(2), 63-72. 